\title{
Phase Structure of Strong-Field Tunneling Wave Packets from Molecules
}

\author{
Ming-Ming Liu, ${ }^{1}$ Min Li, ${ }^{4}$ Chengyin Wu, ${ }^{1,2}$ Qihuang Gong, ${ }^{1,2}$ André Staudte, ${ }^{3}$ and Yunquan Liu ${ }^{1,2, *}$ \\ ${ }^{1}$ Department of Physics and State Key Laboratory for Mesoscopic Physics, Peking University, Beijing 100871, China \\ ${ }^{2}$ Collaborative Innovation Center of Quantum Matter, Beijing 100871, China \\ ${ }^{3}$ Joint Laboratory for Attosecond Science, National Research Council and University of Ottawa, 100 Sussex Drive, \\ Ottawa, Ontario K1A OR6, Canada \\ ${ }^{4}$ School of Physics and Wuhan National Laboratory for Optoelectronics, Huazhong University of Science and Technology, \\ Wuhan 430074, China
}

(Received 7 December 2015; published 20 April 2016)

\begin{abstract}
We study the phase structure of the tunneling wave packets from strong-field ionization of molecules and present a molecular quantum-trajectory Monte Carlo model to describe the laser-driven dynamics of photoelectron momentum distributions of molecules. Using our model, we reproduce and explain the alignment-dependent molecular frame photoelectron spectra of strong-field tunneling ionization of $\mathrm{N}_{2}$ reported by M. Meckel et al. [Nat. Phys. 10, 594 (2014)]. In addition to modeling the low-energy photoelectron angular distributions quantitatively, we extract the phase structure of strong-field molecular tunneling wave packets, shedding light on its physical origin. The initial phase of the tunneling wave packets at the tunnel exit depends on both the initial transverse momentum distribution and the molecular internuclear distance. We further show that the ionizing molecular orbital has a critical effect on the initial phase of the tunneling wave packets. The phase structure of the photoelectron wave packet is a key ingredient for modeling strong-field molecular photoelectron holography, high-harmonic generation, and molecular orbital imaging.
\end{abstract}

DOI: 10.1103/PhysRevLett.116.163004

Optical tunnel ionization is one of the most prominent processes of atoms and molecules interacting with strong laser fields [1]. Following tunneling, the subsequent recollision assisted by the laser fields has triggered many other interesting highly nonlinear processes, e.g., high-harmonic generation $[2,3]$ and nonsequential double ionization $[4,5]$. Both field-assisted tunneling and rescattering form the basis of powerful novel techniques of atomic-resolution imaging for molecular structures and dynamics [6-12]. One of these techniques is strong-field photoelectron holography [11]. Recently, using differential strong-field photoelectron holography, a subtle yet important effect in the tunnel ionization of molecules was discovered. Meckel et al. [13] observed in experiments that the hologram depends on the angle between the laser field and the molecular axis. Solving the 2D time-dependent Schrödinger equation, it was found that the tunnel-ionized electron can be transversely displaced with respect to the molecule's center, depending on the molecular alignment. Consequently, also the recolliding wave packet can be spatially offset in phase and amplitude with respect to the molecular center as revealed by the 3D time-dependent resolution-in-ionic-states (TD-RIS) calculation [13]. Since the phase structure of the tunneling wave packet is critical for all direct ionization and rescattering phenomena, it is very desirable to obtain a qualitative and transparent model that can account for this effect.

Here, we present a robust molecular quantum-trajectory Monte Carlo (MO-QTMC) model to study molecular frame photoelectron angular distributions (MFPADs) in strong laser fields. Using the calculated MFPADs for different alignments between the laser field and the molecular axis, we create a differential hologram. The simulations reproduce the experiment [13] for $\mathrm{N}_{2}$ molecules very well. We further show that the electron wave packets exhibit significant phase distortions after tunneling. Our model provides an intuitive explanation for the distorted phase structure of the molecular tunneling wave packets. Finally, we have applied our model to a molecule with a different electronic structure, i.e., $\mathrm{O}_{2}$, and predict a very different hologram compared to $\mathrm{N}_{2}$.

The QTMC model has been developed to explain photoelectron angular distribution of above threshold ionization of atoms $[14,15]$. In order to study strong-field molecular tunneling ionization, the effect of the molecular orbitals on the tunnel-ionized wave packets should be considered. To obtain the initial structure of the tunneling wave packet in molecules, we start from the transition amplitude from a ground state to a continuum state within the strong-field approximation, which can be expressed as $[16,17]$,

$$
M_{\mathbf{P}}=-i \int_{0}^{T_{p}} d t\left\langle\mathbf{p}+\mathbf{A}(t)|\mathbf{r} \cdot \mathbf{E}(t)| \varphi_{0}\right\rangle e^{i S(t)},
$$

where $\left|\varphi_{0}\right\rangle$ is the ground state of molecule, $\mathbf{E}(t)$ is the electric field, $T_{p}$ is the duration of laser pulse, $\mathbf{p}$ is the drift momentum, and $S(t)=\frac{1}{2} \int{ }^{t} d t^{\prime}\left[\mathbf{p}+\mathbf{A}\left(t^{\prime}\right)\right]^{2}+I_{p} t$ is the 
classical action. Since $[\mathbf{r} \cdot \mathbf{E}(t)+i(\partial / \partial t)] \exp \{-i[\mathbf{p}+$ $\mathbf{A}(t)] \cdot \mathbf{r}\}=0$, one can integrate by parts to obtain,

$$
\begin{aligned}
M_{\mathbf{p}}= & \int_{0}^{T_{p}} d t \tilde{\psi}_{0}(\mathbf{p}+\mathbf{A}(t)) S^{\prime}(t) e^{i S(t)} \\
& -\left.\tilde{\psi}_{0}(\mathbf{p}+\mathbf{A}(t)) e^{i S(t)}\right|_{0} ^{T_{p}}
\end{aligned}
$$

where $\tilde{\psi}_{0}(\mathbf{p})$ is the Fourier transform of the ground state wave function and $S^{\prime}(t)=d S(t) / d t$. For simplicity, we consider the symmetric superposition of $1 \mathrm{~s}$ orbitals to mimic the highest occupied molecular orbital (HOMO) of $\mathrm{N}_{2}[18,19]$,

$$
\tilde{\psi}_{N_{2}}\left(\mathbf{k}, \mathbf{R}_{0}\right)=\frac{2^{5 / 2} \kappa^{5 / 2}}{\pi\left(k^{2}+\kappa^{2}\right)^{2}} \frac{\cos \left(\mathbf{k} \cdot \mathbf{R}_{0} / 2\right)}{\sqrt{2\left[1+S_{1 s}\left(R_{0}\right)\right]}} .
$$

Here, $\mathbf{R}_{0}$ is the vector pointing from one atomic nucleus to the other, $\kappa=\sqrt{2 I_{p}}, \mathbf{k}=\mathbf{p}+\mathbf{A}(t)$, and $S_{1 s}\left(R_{0}\right)$ is the atomic orbital overlap integral. Combining Eq. (2) with Eq. (3), we can rewrite the transition amplitude of Eq. (2) as

$$
M_{\mathbf{p}}=I-\left.\tilde{\psi}_{0}(\mathbf{p}+\mathbf{A}(t)) e^{i S(t)}\right|_{0} ^{T_{p}},
$$

where

$$
I=\frac{\kappa^{5 / 2}}{\pi \sqrt{1+S_{1 s}\left(R_{0}\right)}} \int_{0}^{T_{p}} \cos \left\{\frac{[\mathbf{p}+\mathbf{A}(t)] \cdot \mathbf{R}_{0}}{2}\right\} \frac{e^{i S}}{S^{\prime}} d t .
$$

The main contribution to the integral in Eq. (5) comes from the vicinities of two end points $t=0$ and $t=T_{p}$, and the vicinities of saddle points $t=t_{s}$, which satisfy the saddle point equation i.e., $\left[\mathbf{p}+\mathbf{A}\left(t_{s}\right)\right]^{2}+2 I_{p}=0$. The integral over two end points will counteract the second term on the right-hand side of Eq. (4) [17]. As a result, we need only to consider the integral over the saddle points. Because the term $\cos \left\{[\mathbf{p}+\mathbf{A}(t)] \cdot \mathbf{R}_{0} / 2\right\}$ changes slowly in the vicinities of saddle points, it can be taken out of the integral. Then, after integrating along the steepest descent direction of the saddle points [19], we can obtain the transition amplitude for molecules,

$$
M_{\mathbf{p}}=\frac{i \kappa^{5 / 2}}{\sqrt{1+S_{1 s}\left(R_{0}\right)}} \sum_{s} \cos \left\{\frac{\left[\mathbf{p}+\mathbf{A}\left(t_{s}\right)\right] \cdot \mathbf{R}_{0}}{2}\right\} \frac{e^{i S_{s}}}{S_{s}^{\prime \prime}} .
$$

The molecular structural factor is now incorporated into the transition amplitude with the saddle point approximation. Compared with the atomic case, the molecular transition amplitude is equivalent to the atomic counterpart multiplied by a structural factor. To see how this structural factor modifies the tunneling process, we calculate the transition amplitude based on the saddle point equation. We assume that the laser pulse is linearly polarized and the vector potential is given by $\mathbf{A}(t)=A_{0} \cos (\omega t) \mathbf{x}$ ( $x$ is the polarization direction and $y$ is the transverse direction). Because the momentum at the tunnel exit is related to the drift momentum $\mathbf{p}$ by $\mathbf{v}=\mathbf{p}+\mathbf{A}\left(t_{r}\right)$ [20,21], where $\mathbf{v}$ is the momentum at the tunnel exit and $t_{r}$ is the real part of the saddle point time $t_{s}=t_{r}+i t_{i}$, the saddle point equation can be decomposed into the real and imaginary part [22],

$$
\begin{aligned}
p_{x}+A_{0} \cos \left(\omega t_{r}\right) \cosh \left(-\omega t_{i}\right) & =0 \\
A_{0} \sin \left(\omega t_{r}\right) \sinh \left(-\omega t_{i}\right) & = \pm \sqrt{2 I_{p}+p_{y}^{2}} .
\end{aligned}
$$

Using the expression of vector potential $\mathbf{A}\left(t_{s}\right)$ and Eq. (7), the structural factor can be rewritten as $\cos \left\{\left[\mathbf{p}+\mathbf{A}\left(t_{s}\right)\right]\right.$. $\left.\mathbf{R}_{0} / 2\right\}=\cos \left[p_{y} R_{0} \sin \theta / 2 \pm i R_{0} \cos \theta \sqrt{2 I_{p}+p_{y}^{2}} / 2\right]$, where $\theta$ is the alignment angle of molecules. Using the relation $\cos \left\{\left[\mathbf{p}+\mathbf{A}\left(t_{s}\right)\right] \cdot \mathbf{R}_{0} / 2\right\}=a e^{i \phi}$, one obtains

$$
\begin{aligned}
\tan \phi= & \tan \left(\frac{p_{y} R_{0} \sin \theta}{2}\right) \tanh \left(\mp \frac{R_{0} \cos \theta}{2} \sqrt{2 I_{p}+p_{y}^{2}}\right) \\
a^{2}= & {\left[\cos \left(\frac{p_{y} R_{0} \sin \theta}{2}\right) \cosh \left(\mp \frac{R_{0} \cos \theta}{2} \sqrt{2 I_{p}+p_{y}^{2}}\right)\right]^{2} } \\
& +\left[\sin \left(\frac{p_{y} R_{0} \sin \theta}{2}\right) \sinh \left(\mp \frac{R_{0} \cos \theta}{2} \sqrt{2 I_{p}+p_{y}^{2}}\right)\right]^{2},
\end{aligned}
$$

where $\phi$ is the initial phase of each trajectory and $a^{2}$ is the correction to the ionization rate. One can see that the molecular structure factor depends on the initial transverse momentum $p_{y}$ and the molecular alignment angle $\theta$. Because the structural factor is independent of the laser field, one can treat it as a correction to atomic tunneling theory in the quasistatic approximation $[14,23,24]$.

In order to study the photoelectron angular distributions of aligned $\mathrm{N}_{2}$ in strong laser fields, we have further incorporated above derivations into molecular quantumtrajectory Monte Carlo model (MO-QTMC). Briefly, according to the path integral formulation of quantum mechanics, the evolution of the wave packets after tunneling can be represented by quantum trajectories. The position of the tunnel exit is given by the Landau-effective theory [25]. The tunneled electrons have a Gaussian-like distribution on the transverse momentum perpendicular to the instantaneous laser field and zero longitudinal momentum along the instantaneous laser field at the tunnel exit $[15,23]$. The electron trajectory is weighted by the modified Ammosov-Delone-Krainov (ADK) ionization rate [15,23] as $W\left(t_{0}, p_{y}\right)=a^{2} W_{0}\left(t_{0}\right) W_{\perp}\left(p_{y}\right)$, where $a^{2}$ is the modification factor of ionization rate given by Eq. (8), $W_{\perp}\left(p_{y}\right) \propto$ $\left[\sqrt{2 I_{p}} /\left|E\left(t_{0}\right)\right|\right] \exp \left[-\sqrt{2 I_{p}} p_{y}^{2} /\left|E\left(t_{0}\right)\right|\right]$ depends on the distribution of the initial transverse momentum $p_{y}$, 
and $\left.\quad W_{0}\left(t_{0}\right) \propto\left|\left(2 I_{p}\right)^{2} /\right| E\left(t_{0}\right)\right|^{2 / \sqrt{2 I_{p}}-1} \exp \left[-2\left(2 I_{p}\right)^{3 / 2} /\right.$ $\left.\left|3 E\left(t_{0}\right)\right|\right]$ depends on the instantaneous laser field when the electron is released. After tunneling, the electron motion in the combined laser and Coulomb fields is governed by the Newtonian equations of motion, $\ddot{\mathbf{r}}=-\left(\mathbf{r}-\mathbf{R}_{0} / 2\right) /\left(2\left|\mathbf{r}-\mathbf{R}_{0} / 2\right|^{3}\right)-\left(\mathbf{r}+\mathbf{R}_{0} / 2\right) /\left(2 \mid \mathbf{r}+\mathbf{R}_{0} /\right.$ $\left.\left.2\right|^{3}\right)-\mathbf{E}(t)$. Here, we have included the effect of the molecular potential on the electron trajectories. The electron rescattering process is naturally included when solving the Newtonian equations. The phase of each quantum trajectory is then given by $\phi+S$, where $\phi$ is the initial phase given by Eq. (8) and $S$ is the classical action after tunneling, given by $S=-\int_{t_{r}}^{\infty}\left[\mathbf{v}(t)^{2} / 2-\left(2 \mid \mathbf{r}-\mathbf{R}_{0} /\right.\right.$ $\left.2 \mid)^{-1}-\left(2\left|\mathbf{r}+\mathbf{R}_{0} / 2\right|\right)^{-1}+I_{p}\right] d t$. We sampled the electron ensemble with the Monte Carlo method. The photoelectron angular distributions are obtained by considering the interference of all tunneling electrons on a "virtual" detector [14]. The detailed derivation of this model is given in Supplementary Material [26].

In the simulations, the laser field $\mathbf{E}(t)$ with a cosine waveform has a constant amplitude for the first four cycles and is turned off with a two-cycle ramp. The laser peak intensity is chosen to be $I_{0}=1.3 \times 10^{14} \mathrm{~W} / \mathrm{cm}^{2}$, as the experiment [13]. The simulated momentum distributions at the alignment angles $45^{\circ}$ and $90^{\circ}$ are shown in Figs. 1(a) and 1(b), respectively. We considered the focal volume effect and calculated 12 intensities in the range of $0.7-1.3 \times$ $10^{14} \mathrm{~W} / \mathrm{cm}^{2}$ for each alignment angle. The relative weight of each intensity is given by $d V / d t \propto\left(I_{0}+\right.$ 2I) $\sqrt{I_{0}-I} / I^{5 / 2}$ [27]. One can see the obvious spiderlike structures for all the alignment angles, which comes from the holographic interference between the rescattering and nonscattering electron wave packets [11]. At the first glance, the holographic pattern exhibits the same horizontal fringelike structures for different alignment angles. Indeed, a minor difference can be identified with careful examination. The holographic interference is symmetric about $p_{y}=0$ when the alignment angle is $90^{\circ}$, while the one in the $45^{\circ}$ case is slightly tilted and has an asymmetric distribution about $p_{y}=0$.
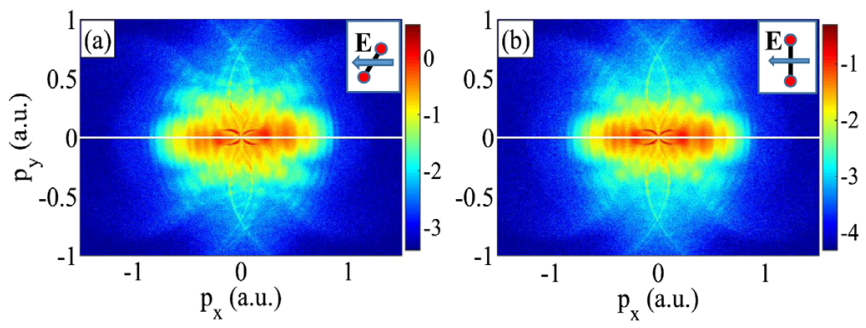

FIG. 1. Calculated photoelectron angular distributions of $\mathrm{N}_{2}$ at the alignment angle of $45^{\circ}$ (a) and $90^{\circ}$ (b), respectively. We considered the focal volume effect and calculated 12 intensities in the range of $0.7-1.3 \times 10^{14} \mathrm{~W} / \mathrm{cm}^{2}$ for each alignment angle. Figures are plotted in logarithmic color scale.
To isolate the alignment dependence of the holographic interference, we now create a differential hologram, following Ref. [13]. For the differential hologram, we calculate the normalized difference $\left[D(\mathbf{p}, \theta)-D_{\text {ref }}(\mathbf{p})\right] /$ $\left[D(\mathbf{p}, \theta)+D_{\text {ref }}(\mathbf{p})\right]$, where $D(\mathbf{p}, \theta)$ is the photoelectron distribution corresponding to the alignment angle $\theta$, the reference $D_{\text {ref }}(\mathbf{p})$ is the sum spectrum over all momentum distributions for alignment angles from $0^{\circ}$ to $180^{\circ}$ in $2^{\circ}$ steps. Both $D(\mathbf{p}, \theta)$ and $D_{\text {ref }}(\mathbf{p})$ are normalized. Note, that the focal volume effect is considered for the normalized momentum difference plots of each alignment angle.

In Fig. 2, we compare our simulated differential holograms (left column) with the experimental ones (right column) in $\mathrm{N}_{2}$. For parallel and perpendicular alignment (top and bottom row), the photoelectron distributions are symmetric with respect to the laser polarization. Hence, the corresponding differential holograms are also symmetric and show no fringes in Figs. 2(a) and 2(c). At the alignment angle $\theta$ of $45^{\circ}$, the holographic interference is asymmetric, as shown in Fig. 2(c). The simulated distributions agree perfectly with the experimental measurement Figs. 2(d)-2(f) (data are taken from Ref. [13]).

In order to understand the puzzling asymmetric distribution at the certain alignment angle, we will investigate the phase structure of strong-field tunneling wave packets and its influence on photoelectron angular distributions of the aligned molecules. In contrast to the tunneling ionization of atoms, the initial phase $\phi$ of molecular tunneling
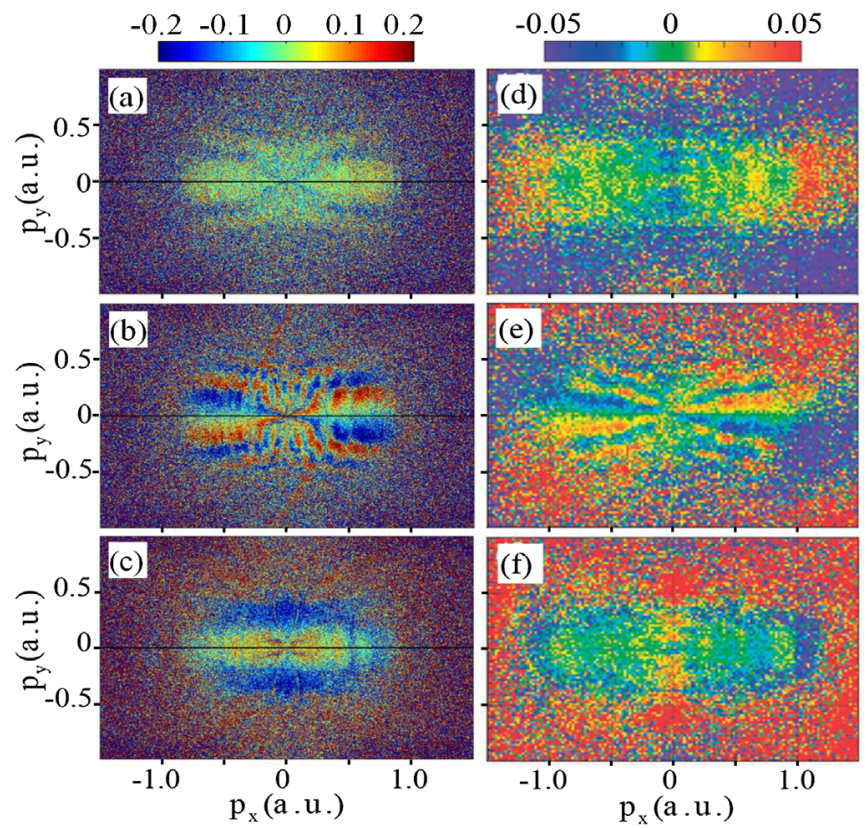

FIG. 2. (a), (b), and (c) are the simulated normalized momentum difference plots at the alignment angle of $0^{\circ}, 45^{\circ}$, and $90^{\circ}$. The ionizing laser pulse polarization is horizontal in all figures. We considered the focal volume effect for all spectra. The right column indicates the corresponding experimental measurements [13]. 
wave packet of $\mathrm{N}_{2}$ is given by Eq. (8), which depends on the internuclear distance as well as the initial transverse momentum at the tunnel exit. In Figs. 3(a) and 3(b), we show the calculated initial phase with respect to the initial transverse momentum for different alignment angles at $R_{0}=2.07$ a.u. and $R_{0}=4$ a.u., respectively.

As seen in Figs. 3(a) and 3(b), in the case of $0^{\circ}$ and $90^{\circ}$, the initial phase is constant at 0 and $\pi$, respectively. In contrast, in the case of an oblique alignment, the initial phase will increase with the transverse momentum, and the slopes are different for different alignment angles. The phase jump for the perpendicular alignment in Fig. 3(b) near $\left|p_{y}\right| \sim 0.8$ a.u. arises from the interference between the contributions from two atomic centers, which depends on the internuclear distance as seen in Eq. (8). However, because the ionization rate decreases substantially at large transverse momentum [indicated with the dashed line with Gaussian distribution in Fig. 3(b)], the phase distortion at larger transverse momentum $\left(\left|p_{y}\right|>0.5\right.$ a.u.) makes a less evident contribution to the deflection of photoelectron angular distributions. At the equilibrium internuclear distance $R_{0}=2.07$ a.u., the phase at the tunneling exit changes very slowly with the initial transverse momentum, and thus is difficult to observe in the photoelectron angular distributions. Note, the initial phase in momentum space originates from the spatial offset of the tunnel ionized wave packet, and the dependence of the initial phase on the transverse momentum at the tunnel exit is the main factor producing the fringelike structure in the normalized momentum difference plots.

We further calculated the photoelectron angular distributions for $\mathrm{O}_{2}$ at different alignment angles. The Fourier transform of the wave function of the $\mathrm{HOMO}$ of $\mathrm{O}_{2}$ is given by [19],

$$
\tilde{\psi}_{O_{2}}\left(\mathbf{k}, \mathbf{R}_{0}\right)=\frac{2^{4} \kappa^{7 / 2} k \sin \theta_{\mathbf{k}} \sin \varphi_{\mathbf{k}}}{\pi\left(k^{2}+\kappa^{2}\right)^{3 / 2}} \frac{\sin \left(\mathbf{k} \cdot \mathbf{R}_{0} / 2\right)}{\sqrt{1-S_{2 p y}\left(R_{0}\right)}} .
$$

Here, $\theta_{\mathbf{k}}$ and $\varphi_{\mathbf{k}}$ are the polar and azimuthal angle of momentum $\mathbf{k}$ with respect to molecular axis $\mathbf{R}_{0}$, and
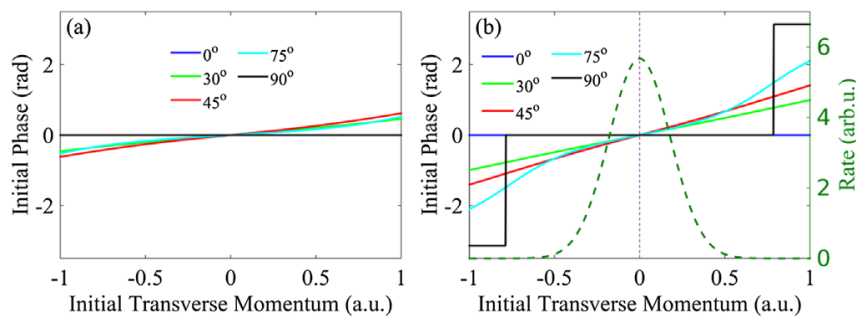

FIG. 3. The dependence of the initial phase $\phi$ given by Eq. (8) on the transverse momentum at the tunnel exit for (a) $R_{0}=$ 2.07 a.u. and (b) $\mathrm{R}_{0}=4$ a.u. for $\mathrm{N}_{2}$. The modified $A D K$ ionization rate with respect to the initial transverse momentum at the alignment angle of $45^{\circ}$ is illustrated as the dashed curve in Fig. 3(b).
$S_{2 p_{y}}\left(R_{0}\right)$ is the atomic orbital overlap integral. Following a similar derivation in $\mathrm{N}_{2}$, we simulate the photoelectron angular distributions for $\mathrm{O}_{2}\left(R_{0}=4\right.$ a.u. $)$ at the alignment angle $0^{\circ}, 45^{\circ}$, and $90^{\circ}$ with respect to the laser polarization at an laser intensity of $I_{0}=1.3 \times 10^{14} \mathrm{~W} / \mathrm{cm}^{2}$, as shown in Figs. 4(a)-4(c). We can see that the photoelectron angular distributions at the alignment angles of $0^{\circ}$ and $90^{\circ}$ exhibit a minimum along the polarization axis, which originates from the node structure in the $\mathrm{HOMO}$ of $\mathrm{O}_{2}$. This minimum has been observed for $\mathrm{O}_{2}$ experimentally [28]. It was explained using molecular-strong-field approximation (MO-SFA) model [29], where the rescattering effect dominates. In contrast, direct tunneling ionization dominates the low-energy photoelectron momentum distributions, and the minimum observed in the momentum distributions is the direct manifestation of molecular node structure.

In Fig. 4(d), we plot the initial phase with respect to the initial transverse momentum for $\mathrm{O}_{2}$. Similar to the case of $\mathrm{N}_{2}$, the initial phase at the alignment angle of $0^{\circ}$ and $90^{\circ}$ is a constant. However, there is a phase jump of $\pi$ at zero momentum for the parallel and perpendicular alignment. The phase jump reflects the node structure in the HOMO of $\mathrm{O}_{2}$. Since the outermost orbital of $\mathrm{O}_{2}$ is $\pi_{g}$ geometry, the calculated differential hologram is remarkably different to $\mathrm{N}_{2}$, as seen in Fig. 5. At the alignment angle $0^{\circ}$ and $90^{\circ}$, the distinct minimum along the laser polarization axis can be clearly observed in the differential holograms.

In summary, we have developed a MO-QTMC model to describe the laser-driven dynamics of electron tunneling process in molecules. The study elucidated that the asymmetrical distribution in differential holograms originates from the initial phase structure of the tunneling wave packets in the momentum representation for $\mathrm{N}_{2}$. We showed that the initial phase of the continuum wave packets can be extracted from the outermost molecular
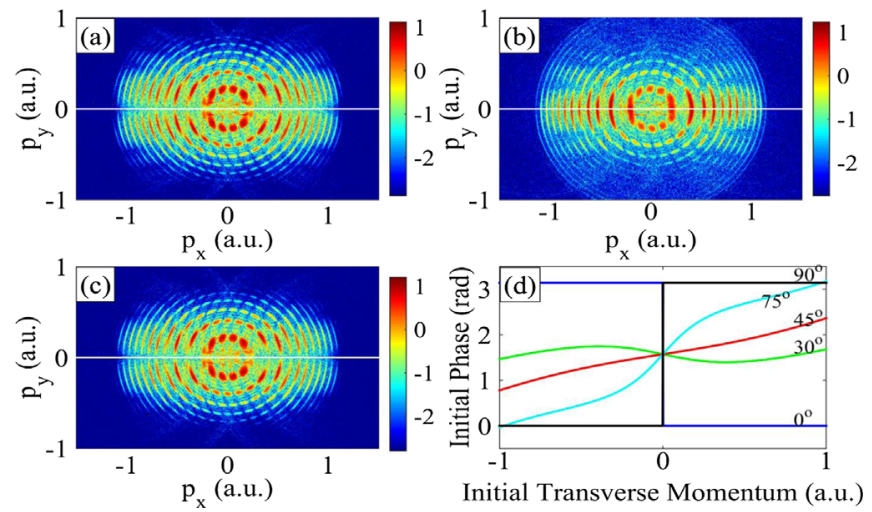

FIG. 4. (a), (b), and (c) are the calculated photoelectron angular distributions of $\mathrm{O}_{2}$ at the alignment angle $0^{\circ}, 45^{\circ}$, and $90^{\circ}$, respectively. The laser parameters of the simulation are the same as those of $\mathrm{N}_{2}$. The focal volume effect is not included in the plots. Figures are plotted in logarithmic color scale. (d) shows the relation between the initial phase $\phi$ and the transverse momentum at the tunnel exit for $\mathrm{O}_{2}$. 


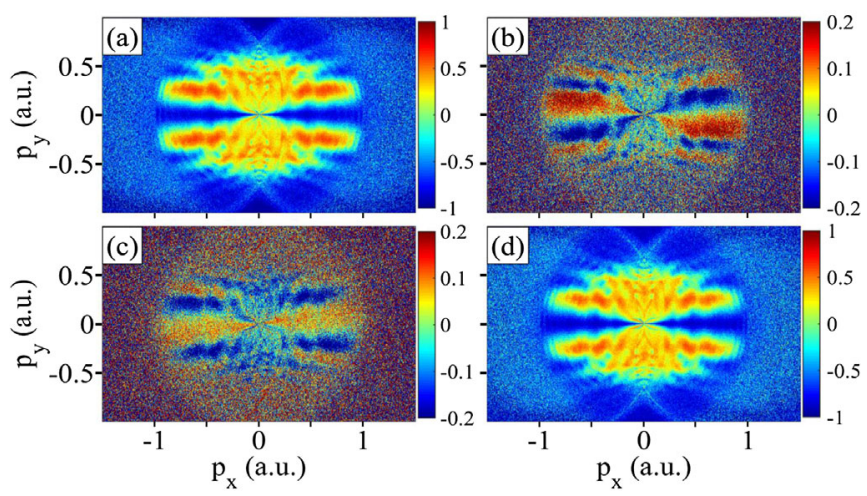

FIG. 5. The simulated differential holograms for $\mathrm{O}_{2}$ at the alignment angle of (a) $0^{\circ}$, (b) $22^{\circ}$, (c) $44^{\circ}$, and (d) $90^{\circ}$. We considered the focal volume effect for the normalized momentum spectra. The peak intensity is $I_{0}=1.3 \times 10^{14} \mathrm{~W} / \mathrm{cm}^{2}$.

orbital structure and the alignment geometry with respect to the field polarization. Including the phase structure in the semiclassical model, the subtle structure of photoelectron holography of molecules using strong laser fields can be illuminated. Since the tunneling process is predominant in the strong-field community, this study has important implications for the physics and applications of the laser-driven tunneling ionization and laser-driven recollision of molecules. The MO-QTMC approach can be further extended for strong-field ionization of polyatomic molecules.

We thank Professor C.-D. Lin for instructive discussions. This work is supported by the National Program on Key Basic Research Project (Grant No. 2013CB922403) and the National Science Foundation of China (No. 11434002 and No. 11125416).

\section{*Yunquan.liu@pku.edu.cn}

[1] L. V. Keldysh, Zh. Eksp. Teor. Fiz. 47, 1945 (1964) [Sov. Phys. JETP 20, 1307 (1965)].

[2] A. L'Huillier and Ph. Balcou, Phys. Rev. Lett. 70, 774 (1993).

[3] J. L. Krause, K. J. Schafer, and K. C. Kulander, Phys. Rev. Lett. 68, 3535 (1992).

[4] D. N. Fittinghoff, P. R. Bolton, B. Chang, and K. C. Kulander, Phys. Rev. Lett. 69, 2642 (1992).

[5] B. Walker, B. Sheehy, L. F. DiMauro, P. Agostini, K. J. Schafer, and K. C. Kulander, Phys. Rev. Lett. 73, 1227 (1994).

[6] H. Akagi, T. Otobe, A. Staudte, A. Shiner, F. Turner, R. Dörner, D. M. Villeneuve, and P. B. Corkum, Science 325, 1364 (2009).
[7] H. Liu, S.-F. Zhao, M. Li, Y. Deng, C. Wu, X.-X. Zhou, Q. Gong, and Y. Liu, Phys. Rev. A 88, 061401(R) (2013).

[8] M. Meckel, D. Comtois, D. Zeidler, A. Staudte, D. Pavičić, H. C. Bandulet, H. Pépin, J. C. Kieffer, R. Dörner, D. M. Villeneuve, and P. B. Corkum, Science 320, 1478 (2008).

[9] C. I. Blaga, J. Xu, A. D. Dichiara, E. Sistrunk, L. Zhang, P. Agostini, T. A. Miller, L. F. Dimauro, and C. D. Lin, Nature (London) 483, 194 (2012).

[10] J. Itatani, J. Levesque, D. Zeidler, H. Niikura, H. Pépin, J. C. Kieffer, P. B. Corkum, and D. M. Villeneuve, Nature (London) 432, 867 (2004).

[11] Y. Huismans et al., Science 331, 61 (2011).

[12] M. Li, X. Sun, X. Xie, Y. Shao, Y. Deng, C. Wu, Q. Gong, and Y. Liu, Sci. Rep. 5, 8519 (2015).

[13] M. Meckel, A. Staudte, S. Patchkovskii, D. M. Villeneuve, P. B. Corkum, R. Dörner, and M. Spanner, Nat. Phys. 10, 594 (2014).

[14] M. Li, J.-W. Geng, H. Liu, Y. Deng, C. Wu, L.-Y. Peng, Q. Gong, and Y. Liu, Phys. Rev. Lett. 112, 113002 (2014).

[15] M. Li, J.-W. Geng, M. Han, M. M. Liu, L.-Y. Peng, Q. Gong, and Y. Liu, Phys. Rev. A 93, 013402 (2016).

[16] W. Becker, F. Grasbon, R. Kopold, D. B. Milošević, G. G. Paulus, and H. Walther, Adv. At. Mol. Opt. Phys. 48, 35 (2002).

[17] D. B. Milošević, G. G. Paulus, D. Bauer, and W. Becker, J. Phys. B 39, R203 (2006).

[18] J. Muth-Böhm, A. Becker, and F. H. M. Faisal, Phys. Rev. Lett. 85, 2280 (2000).

[19] V. I. Usachenko and S.-I. Chu, Phys. Rev. A 71, 063410 (2005).

[20] M. Li, J.-W. Geng, M. M. Liu, X. Zheng, L.-Y. Peng, Q. Gong, and Y. Liu, Phys. Rev. A 92, 013416 (2015).

[21] T.-M. Yan and D. Bauer, Phys. Rev. A 86, 053403 (2012).

[22] The " \pm " sign in Eq. (7) is "+" when the electric field is negative and is "-" when the electric field is positive. The sign in Eq. (8) is the same as the sign of the electric field.

[23] N. B. Delone and V. P. Krainov, J. Opt. Soc. Am. B 8, 1207 (1991).

[24] M. V. Ammosov, N. B. Delone, and V. P. Krainov, Sov. Phys. JETP 64, 1191 (1986).

[25] L.D. Landau and E. M. Lifshitz, Quantum Mechanics (Pergamon, Oxford, 1977).

[26] See Supplemental Material at http://link.aps.org/ supplemental/10.1103/PhysRevLett.116.163004 for the detailed description of MO-QTMC model.

[27] S. Augst, D. D. Meyerhofer, D. Strickland, and S. L. Chin, J. Opt. Soc. Am. B 8, 858 (1991).

[28] M. Okunishi, R. Itaya, K. Shimada, G. Prümper, K. Ueda, M. Busuladžić, A. Gazibegović-Busuladžić, D. Milošević, and W. Becker, Phys. Rev. Lett. 103, 043001 (2009).

[29] M. Busuladžić, A. Gazibegović-Busuladžić, D. B. Milošević, and W. Becker, Phys. Rev. Lett. 100, 203003 (2008). 